\title{
Muography of 1949 fault in La Palma, Canary Islands, Spain
}

Seigo Miyamoto ${ }^{1,}$, José Barrancos ${ }^{2,3}$, Cristiano Bozza ${ }^{4}$, Lucia Consiglio ${ }^{5}$, Chiara De Sio ${ }^{4}$, Pedro Hernández $z^{2,3,6}$, Ryuichi Nishiyama ${ }^{1}$, Germán Padilla ${ }^{2,3}$, Eleazar Padrón ${ }^{2,3}$, Chiara Sirignano $^{7,8}$, Simona Maria Stellacci ${ }^{4}$, Hiroyuki K.M. Tanaka ${ }^{1}$, Valeri Tioukov ${ }^{5}$

${ }^{1}$ Earthquake Research Institute, The University of Tokyo, Tokyo, Japan

${ }^{2}$ Instituto Tecnológico y de Energías Renovables (ITER), Santa Cruz de Tenerife, Spain

${ }^{3}$ Instituto Volcanológico de Canarias (INVOLCAN), Puerto de la Cruz, Tenerife, Spain

${ }^{4}$ Università di Salerno, Dipartimento di Fisica "E. R. Caianiello", Salerno, Italy

${ }^{5}$ Università di Napoli Federico II, Dipartimento di Fisica, Naples, Italy

${ }^{6}$ Agencia Insular de Energía de Tenerife (AIET), Granadilla de Abona, Tenerife, Spain

${ }^{7}$ Università di Padova, Dipartimento di Fisica e Astronomia "Galileo Galilei”, Padova, Italy

${ }^{8}$ Istituto Nazionale di Fisica Nucleare (INFN), Sezione di Padova, Padova, Italy

\section{Article history}

Received July 5, 2016; accepted October 5, 2016.

Subject classification:

Muon radiography, Muography, Volcano, Fault, Land slide.

\section{ABSTRACT}

Muography (muon radiography) is a new geophysical technique that allows investigation of inner structures of an edifice with a very detailed spatial resolution. It has been recently used for several volcanoes and different geoscientific targets. In 2011 Tanaka et al. succeeded to find hidden ancient seismic faults. In 1949 there was a volcanic activity of the Cumbre Vieja, La Palma, Canary Islands, Spain and a $1 \mathrm{~km}$-long fault suddenly appeared during the active period. The fault might be the sign of a large scale land collapse. In order to get additional information, investigations by several geophysical exploration techniques are needed. We consider that muography can be applied to study the shallow part of the fault and it can clarify some important parameters: the bulk density, the width of the low density zone, and the depth. Previous investigations allowed detecting two ancient seismic faults that have $20 \mathrm{~m}$-wide mechanically fractured zone consisting of highly damaged rocks. The 1949 fault might be the result of large scale land slide and the slide length might be just a few meter. Therefore the expected width of the fault is only a few meter. In order to detect such narrow fault, the muon detector should have as high spatial resolution as possible. In addition, it is difficult to get continuous power supply near the fault. Nuclear emulsions are a kind of photographic films that have high sensitivity for high energy charged particles. They also have high spatial resolution for high energy muon paths and do not need any power supply to be operated. We placed an emulsion detector having $0.19 \mathrm{~m}^{2} \mathrm{ef}$ fective area near the 1949 fault. The exposure started on January 2014 and lasted 106 days. All the emulsion films were developed and they are under analysis. We also estimated the expected performance of this test exposure. Assuming a very simple model, we evaluated the detectable region as a function of the low density zone width and of the depth from the ground surface as well.

\section{Introduction}

La Palma is a volcanic island located in the northwest sector of the Canary Islands (Figure 1). It spans 45 $\mathrm{km}$ in north-south direction and $28 \mathrm{~km}$ in east-west directions, respectively. The area is $706 \mathrm{~km}^{2}$ and the maximum altitude is $2423 \mathrm{~m}$. La Palma consists of two volcanic systems. The northern part of the island was created by the activity of the older volcano Cumbre Nueva (more than 3.0 Ma; Staudigel et al. [1986]) and the southern part has been built by the activity of Cumbre Vieja until

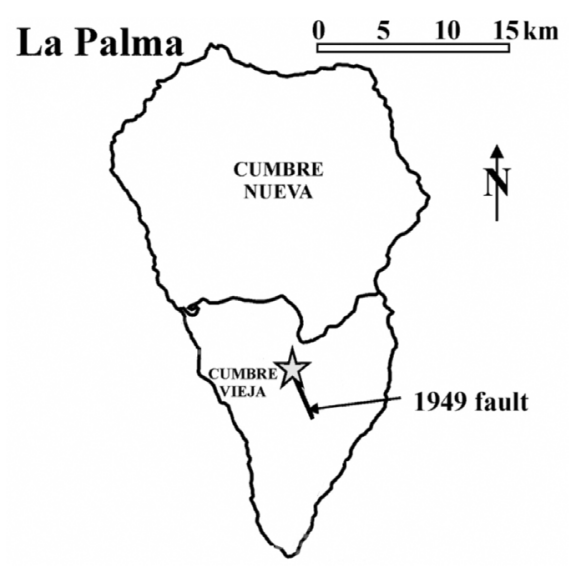

Figure 1. The shape of the La Palma island and the position of 1949 fault. The muon detector installation site is marked by the star. 
$123 \mathrm{ka}$ ago. The length of the main north-south rift zone is about $20 \mathrm{~km}$ [Ancochea et al. 1994, Day et al. 1999].

The collapse of the mountain flank is ordinary for volcanoes and sometimes it can affect very seriously the nearby population. Recent geological investigation shows that the 1949 volcanic eruption near the summit of the Cumbre Vieja might have been accompanied by the development of a west facing fault system along the crest of the volcano. It is considered to be the first surface rupture along a developing zone of flank instability [Day et al. 1999]. This fault is not the surface of a dyke and might be a sign of large scale land collapse. There are many ancient deposits of debris flow and debris avalanches distributed on the seafloor around the Canary Islands and some of them are larger than the area of the island itself [Krastel et al. 2001]. The vertical ground layer structure of La Palma suggests that the weak layer is made of debris avalanche deposit and/ or collapse distributed on the seafloor by the activity of older volcano Cumbre Nueva, and the eruption product of Cumbre Vieja accumulated on it. The magma bulge beneath the north-south rift zone may cause the rupture of the Cumbre Vieja flank with the product of collapse running into the sea at the speed of $100 \mathrm{~m} / \mathrm{s}$. This might generate a huge tsunami and give rise to a very serious
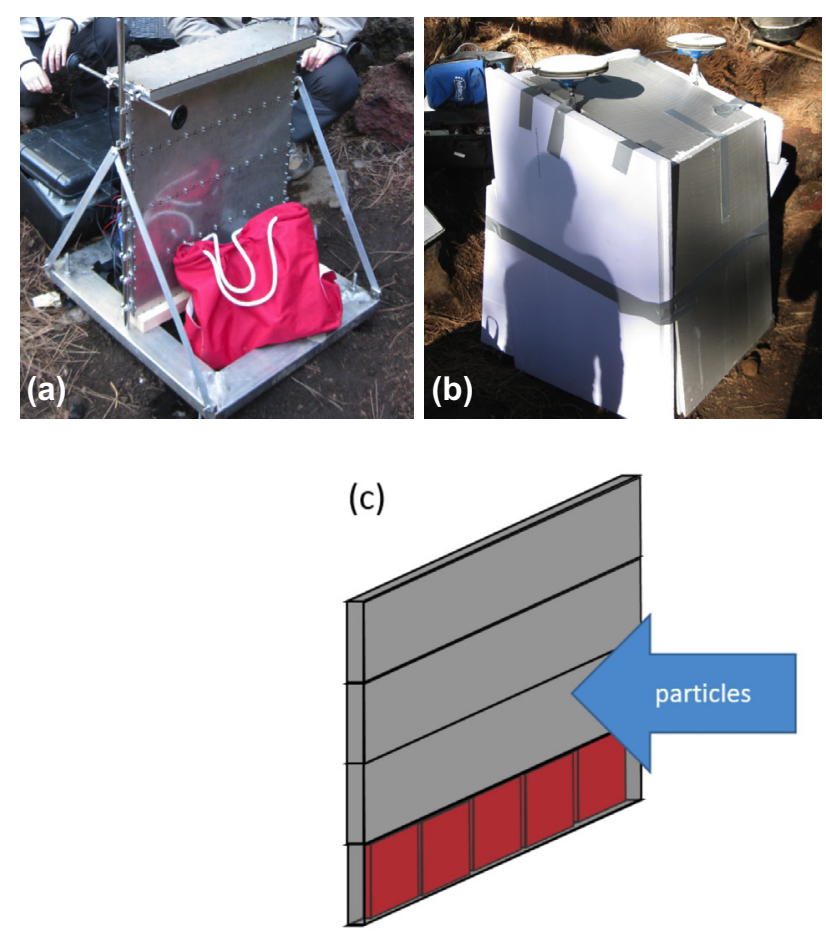

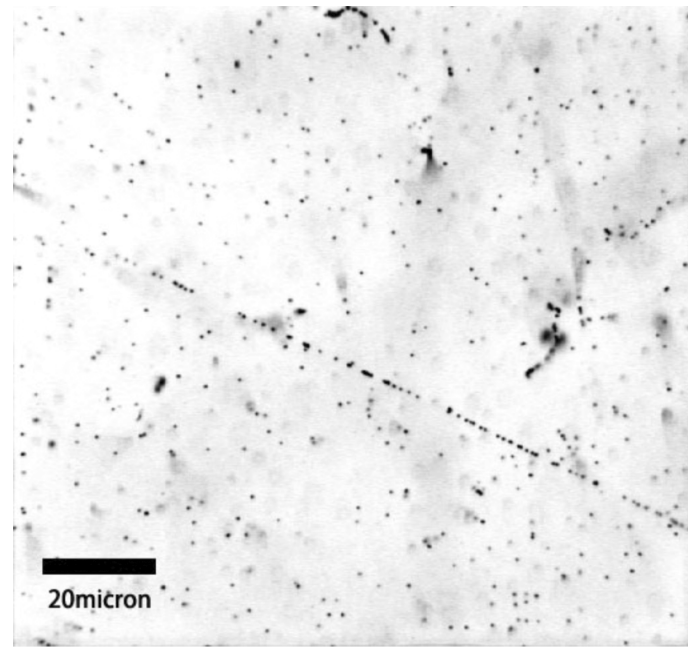

Figure 2. An image of the sensitive layer of nuclear emulsion by using an optical microscope. The size of the view is 120 micron* 105 micron. Each silver grain diameter is about 1 micron and the field depth of the objective is about 3 micron. Aligned grains on the straight line are the track produced by high energy charged minimum ionization particle.

damage to the islands and the north-west coast of Africa and also to the east coast of America [Ward and Day 2001]. It is necessary to investigate the 1949 fault by various geophysical techniques, not only the standard ones, for proper risk estimation.

Cosmic-ray muon radiography (muography) is a new method that can be used to make a map of the inner density structures of large objects such as volcanoes. Many investigations were performed on volcanoes by this method recently [Tanaka et al. 2007a, 2007b, 2007c, 2009a, 2009b, 2010, 2014; Lesparre et al. 2012, Jourde et al. 2013, Cârloganu et al. 2013, Carbone at al. 2013]. The principle is similar to the one of ordinary X-ray photography. The source that corresponds to X-rays is provided by cosmic-rays from the sky, muon

(d)

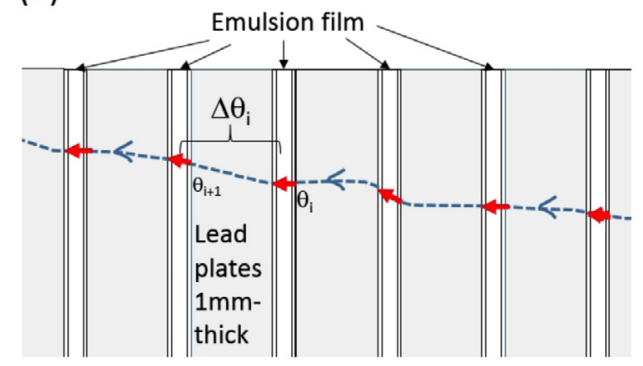

Figure 3. (a) Picture of the rigid metallic frame. ECC made by ten $1 \mathrm{~mm}$-thick lead plates and eleven emulsion films are stacked inside the frame. The arrow shows the direction of the muon emerging from the fault. (b) The rigid frame was covered by polystyrene thick foils to prevent heating and rapid changes of the surrounding temperature. Two GPS antennas were attached to the frame to measure the position and the direction of the detector. (c) The schematic of the muon detector inside. Four metallic frames were stacked in a vertical direction and five ECC were included in each frame. (d) The cross view of each ECC is represented as a schematic diagram. The root mean square of deflection angles $\left(\Delta \theta_{\mathrm{i}}\right)$ of charged particle in the material is proportional to the inverse of momentum and the square root of the radiation length of the material. Considering the angular resolution of emulsion films and the radiation length of the lead plates, most charged particles with momentum below $2 \mathrm{GeV} / \mathrm{c}$ should be rejected. 
detectors act as X-ray films, and the imaging target are volcanoes. The remarkable difference between X-rays and cosmic-ray muons is the attenuation length in the material. The typical attenuation length of $\mathrm{X}$-rays is less than $100 \mathrm{~g} / \mathrm{cm}^{2}$ "density length" ( $1 \mathrm{~m}$ water equivalent), while the attenuation length of cosmic-ray muons ranges from $10 \mathrm{~m}$ to more than $1 \mathrm{~km}$ water equivalent depending on the momentum.

In the previous observation of the low density rock crushing zone in the ancient seismic fault in Itoigawa, Japan, the low density zone width was evaluated to be about $20 \mathrm{~m}$ [Tanaka et al. 2011]. In the case of La Palma, the expected width of the 1949 fault is just a few meters. A muon detector with high spatial resolution is needed. Furthermore, it's difficult to get stable and continuous electric power supply around the 1949 fault. The features of nuclear emulsions (Figure 2) [Nakamura et al. 2006], compared with the ones of any other muon detectors are the following: 1) intrinsic high spatial resolution; 2) no electric power supply needed.

\section{Detector installation and exposure of cosmic-rays}

In January 2014 a rigid metallic frame containing Emulsion Cloud Chamber (ECC) and having $0.19 \mathrm{~m}^{2}$ effective area (Figure 3a) was placed at the bottom of the ravine (Figure 4). ECC has a modular structure made of a sandwich of passive material plates such as lead interleaved with emulsion film layers (Figure 3c). We used ECC to remove low momentum particles by the detection of their large deflection angles in lead (Figure 3d) [Nishiyama et al. 2014]. The detector position and the orientation were measured by two GPS re-

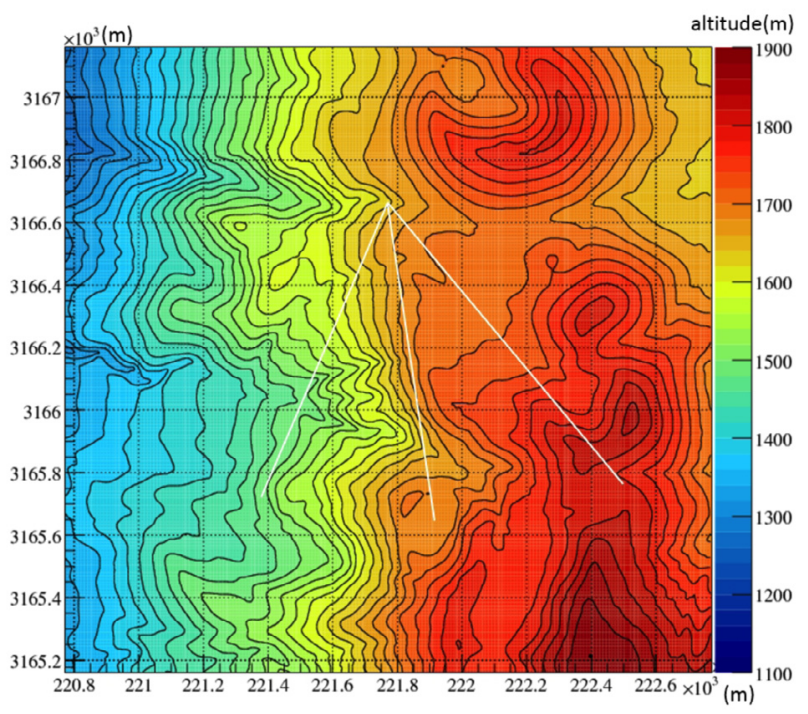

Figure 4. Topological map around the 1949 fault. The color scale represents the altitude and the pitch of the contours is $20 \mathrm{~m}$. The acceptance of the muon detector is indicated by the white solid lines and it is about \pm 30 degree. The vertex is the position of the muon detector. The grid pitch is $200 \mathrm{~m}$

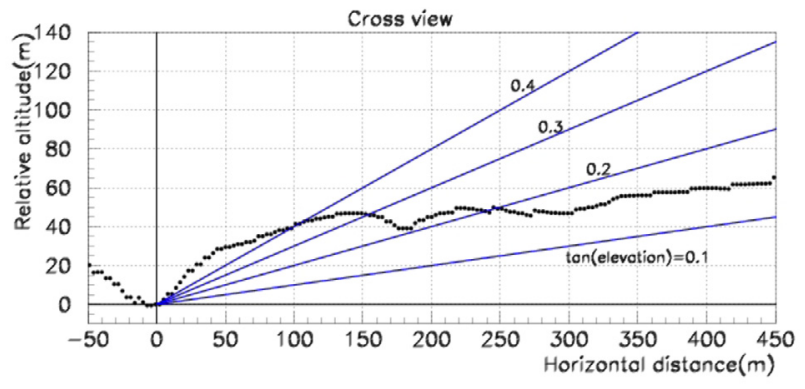

Figure 5. Topographic side view along the fault. Note that the scale is different between horizontal and vertical axis. Blue lines emerging from the detector point indicate the muon paths; they have 0.1 , $0.2,0.3$ and 0.4 tangent of the elevation angle respectively.

ceivers as shown in Figure 3b. After 106 days' exposure, the detector was removed and all films were developed. Now the films are under data taking by automated readout systems [Armenise et al. 2005; Arrabito et al. 2006]. Such systems acquire tomographic images in the emulsion sensitive layers and recognizes straight sequences of silver grains leaved by muons. The output data are the positions and the directions in $\mathrm{x}$-and $\mathrm{y}$-axis, respectively. After the reconstruction of tracks in ECC by offline processing [Tioukov et al. 2006], muon candidates are selected by average number of grains per length and average deflection angle.

\section{Estimation of the expected performance}

We estimated the detectable region in this exposure. The maximum detectable depth from the ground surface is determined by the topography around the fault and the detector. The side view of the topography along the fault is shown in Figure 5. For example, if the elevation angle is $200 \mathrm{mrad}$, the maximum depth from the ground surface is about $20 \mathrm{~m}$, while if elevation angle is $100 \mathrm{mrad}$, the path length is too long to get enough muon statistics to evaluate the density.

The maximum detectable depth also depends on the width of the low density zone in the fault; as the width of the low density zone increases, the solid angle for low density path increases, consequently the available statistics of penetrating muon increases. In Figure 6 the expected detectable region is shown as a function of the width of the low density zone and of the depth from the ground surface. The parameters we assumed are the following: a) effective area is $0.19 \mathrm{~m}^{2}$; b) exposure time is 106 days; c) muon flux model by Honda et al. [2004] and the muon energy attenuation by Groom et al. [2001] are used; d) the muon detection efficiency is $79 \%$ and it has no angular dependence; e) the bulk density of the rock around the fault is uniform (2.2 $\mathrm{g} / \mathrm{cm}^{3}$ ) while a density of $2.0,1.9,1.8,1.7 \mathrm{~g} / \mathrm{cm}^{3}$ in the rock crushing low density zone is supposed. Figure 6 shows our detection limit in this observation if no ex- 


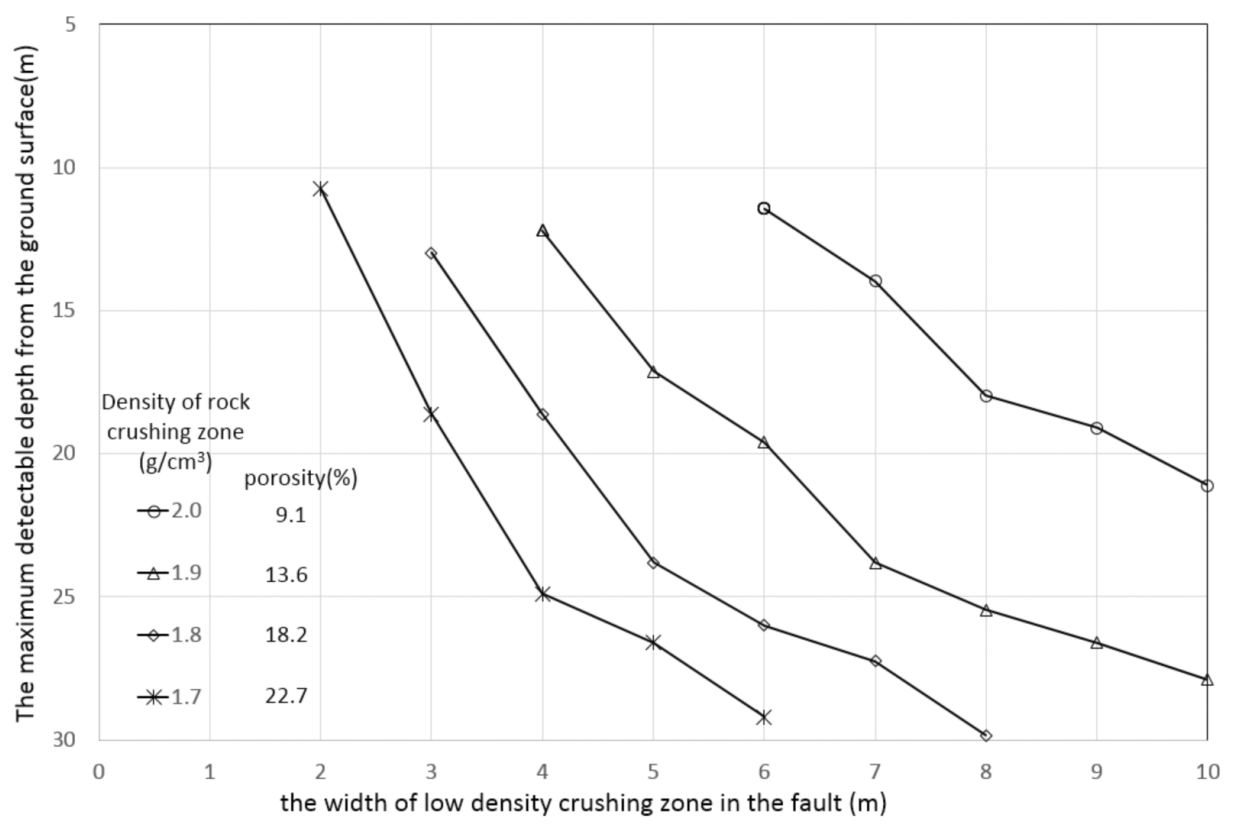

Figure 6. Relation between the width of low density crushing zone and the maximum detectable depth from the ground surface. If both parameters are above the solid line, we can detect the low density zone of the fault with more than $90 \%$ statistical confidence level.

pected contrast region is found. If so, the thickness of the low density zone of crushed rock should be small, less than 2 meter in the case of $1.7 \mathrm{~g} / \mathrm{cm}^{3}$.

\section{Conclusion}

There is the possibility that the 1949 fault in La Palma is the sign of a large scale land collapse. High spatial resolution is necessary to gather information about this fault. We applied the muon radiography method to investigate this fault with a nuclear emulsion based detector. All the films are under analysis. We estimated the detectable region for some important parameters assuming a very simple model. We might see/exclude inner bulk density anomalies in the detectable region at the end of emulsion measurements and data analysis.

Acknowledgements. This research was financed through a grant from the Canary Islands Government Research Agency, ACIISI (ProID20100160), and supported by the Cabildos Insulares de Tenerife and La Palma. We acknowledge the Gran Sasso Laboratory and INFN for their support in the emulsion development activity. We also thank Conny Spelbrink for their logistical support during the fieldwork in Cumbre Vieja.

\section{References}

Ancoechea, E., F. Hernan, A. Cendrero, J.M. Cantragrel, J.M. Fuster, E. Ibarrola and J.Coello (1994). Constructive and destructive episodes in the building of a young Oceanic Island, La Palma, Canary Islands, and genesis of the Caldera de Taburiente, J. Volcanol. Geoth. Res., 60, 243-262.

Armenise, N., et al. (2005). High-speed particle tracking in nuclear emulsion by last-generation automatic microscopes, Nucl. Instrum. Meth. A, 551, 261-270.
Arrabito, L., et al. (2006). Hardware performance of a scanning system for high speed analysis of nuclear emulsions, Nucl. Instrum. Meth. A, 568, 578-587.

Carbone, D., D. Gibert, J. Marteau, M. Diament, L. Zuccarello and E. Galichet (2013). An experiment of muon radiography at Mt Etna (Italy), Geophys. J. Int., 196 (2), 633-643; doi:10.1093/gji/ggt403.

Cârloganu, C., et al. (2013). Towards a muon radiography of the Puy de Dôme, Geosci. Instrum. Meth., 2 (1), 55-60.

Day, S.J., J.C. Carracedo, H. Guillou and P. Gravestock (1999). Recent structural evolution of the Cumbre Vieja volcano, Canary Islands: volcanic rift zones reconfiguration as a precursor to volcano flank instability?, J. Volcanol. Geoth. Res., 94, 135-167.

Krastel, S., H.U. Schmincke, C.L. Jacobs, R. Rihm, T.P. Le Bas and B. Alibes (2001), Submarine landslides around the Canary Islands, J. Geophys. Res., 106 (B3), 3977-3997.

Groom, D.E., N.V. Mokhov and S.I. Striganov (2001). Muon stopping muon stopping power and range tables $10 \mathrm{MeV}-100 \mathrm{TeV}$, Atom. Data Nucl. Data, 78, 183-356.

Honda, M., T. Kajita, K. Kasahara and S. Midorikawa (2004). New calculation of the atmospheric neutrino flux in a three-dimensional scheme, Phys. Rev. D, 70, 043008; doi:10.1103/ PhysRevD.70.043008.

Jourde, K., D. Gibert, J. Marteau, J. De Bremond d'Ars, S. Gardien, C. Girerd, J.-C. Ianigro and D. Carbone (2013). Experimental detection of upward going cosmic particles and consequences for correction of density radiography of volcanoes, Geophys. Res. Lett., 40, 6334-6339; doi:10.1002/2013GL058357. 
Lesparre, N., D. Gibert, J. Marteau, J.C. Komorowski, F. Nicollin and O. Coutant (2012). Density muon radiography of La Soufrière of Guadeloupe volcano: comparison with geological, electrical resistivity and gravity data, Geophys. J. Int., 190, 1008-1019.

Nakamura, T., et al. (2006). The OPERA film: New nuclear emulsion for large-scale, high-precision experiments, Nucl. Instum. Meth. A, 556, 80-86.

Nishiyama, R., S. Miyamoto and N. Naganawa (2014). Experimental study of source of background noise in muon radiography using emulsion film detectors, Geosci. Instrum. Meth., 3, 29-39.

Staudigel, H., G. Feraud and G. Giannerini (1986). The history of intrusive activity on the island of $\mathrm{La}$ Palma (Canary Islands), J. Volcanol. Geoth. Res., 27, 299-322.

Tanaka, H.K.M., T. Nakano, S. Takahashi, J. Yoshida, H. Ohshima, T. Maekawa, H. Watanabe and K. Niwa (2007a). Imaging the conduit size of the dome with cosmic ray muons: The structure beneath Showa Shinzan Lava Dome, Japan, Geophys. Res. Lett., 34, L22311; doi:10.1029/2007GL031389.

Tanaka, H.K.M., T. Nakano, S. Takahashi, J. Yoshida, M. Takeo, J. Oikawa, T. Ohminato, Y. Aoki, E. Koyama, H. Tsuji and K. Niwa (2007b). High resolution imaging in the inhomogeneous crust with cosmic ray muon radiography: The density structure below the volcanic crater floor of Mt. Asama, Japan, Earth Planet. Sc. Lett., 263, 104-113.

Tanaka, H.K.M., T. Nakano, S. Takahashi, J. Yoshida and K. Niwa (2007c). Development of an emulsion imaging system for cosmic-ray muon radiography to explore the internal structure of a volcano, Mt. Asama, Nucl. Instrum. Meth. A, 575, 489-497.

Tanaka, H.K.M., T. Uchida, M. Tanaka and H. Shinohara (2009a). Cosmic-ray muon imaging of magma in a conduit: Degassing process of Satsuma-Iwojima Volcano, Japan, Geophys. Res. Lett., 36, L01304, 1-5.

Tanaka, H.K.M., T. Uchida, M. Tanaka, M. Takeo, J. Oikawa, T. Ohminato, Y. Aoki, E. Koyama and H. Tsuji (2009b). Detecting a mass change inside a volcano by cosmic-ray muon radiography (muography): First results from measurements at Asama volcano, Japan, Geophys. Res. Lett., 36, L17302, 1-4.

Tanaka, H.K.M., H. Taira, T. Uchida, M. Tanaka, M. Takeo, T. Ohminato, Y. Aoki, R. Nishitama, D. Shoji and H. Tsuiji (2010). Three-dimensional computational axial tomography scan of a volcano with cosmic ray muon radiography, J. Geophys. Res., 115, B12332; doi:10.1029/2010JB007677.

Tanaka, H.K.M., H. Miyajima, T. Kusagaya, A. Taketa, T. Uchida and M. Tanaka (2011). Cosmic muon imaging of hidden seismic fault zones: rainwater per- meation into the mechanical fractured zones in Itoigawa-Shizuoka Tectonic Line, Japan, Earth Planet. Sc. Lett., 306, 156-162.

Tanaka, H.K.M., T. Kusagaya and H. Shinohara (2014). Radiographic visualization of magma dynamics in an erupting volcano, Nat. Commun., 5, 3381, 1.

Tioukov, V., I. Kreslo, Y. Petukhov and G. Sirri (2006). The FEDRA-Framework for emulsion data reconstruction and analysis in the OPERA experiment, Nucl. Instrum. Meth. A, 559, 103-105.

Ward, S.N., and S. Day (2001). Cumbre Vieja VolcanoPotential collapse and tsunami at La Palma, Canary Islands, Geophys. Res. Lett., 28, 3397-3400; doi:10.10 29/2001GL013110.

\footnotetext{
${ }^{\star}$ Corresponding author: Seigo Miyamoto, Earthquake Research Institute, The University of Tokyo, Tokyo, Japan; email: miyamoto@eri.u-tokyo.ac.jp.

(C) 2017 by the Istituto Nazionale di Geofisica e Vulcanologia. All rights reserved.
} 